\title{
YAP regulates neural progenitor cell number via the TEA domain transcription factor
}

\author{
Xinwei Cao, ${ }^{1}$ Samuel L. Pfaff, ${ }^{2,4}$ and Fred H. Gage ${ }^{1,3}$ \\ ${ }^{1}$ Laboratory of Genetics, The Salk Institute for Biological Studies, La Jolla, California 92037, USA, ${ }^{2}$ Howard Hughes Medical \\ Institute, Gene Expression Laboratory, The Salk Institute for Biological Studies, La Jolla, California 92037, USA
}

Tight control of cell proliferation is essential for proper growth during development and for tissue homeostasis in mature animals. The evolutionarily conserved Hippo pathway restrains proliferation through a kinase cascade that culminates in the inhibition of the transcriptional coactivator YAP. Unphosphorylated YAP activates genes involved in cell proliferation and survival by interacting with a DNA-binding factor. Here we show that during vertebrate neural tube development, the TEA domain transcription factor (TEAD) is the cognate DNA-binding partner of YAP. YAP and TEAD gain of function causes marked expansion of the neural progenitor population, partly owing to their ability to promote cell cycle progression by inducing cyclin D1 and to inhibit differentiation by suppressing NeuroM. Their loss of function results in increased apoptosis, whereas repressing their target genes leads to premature neuronal differentiation. Inhibiting the upstream kinases of the Hippo pathway also causes neural progenitor overproliferation. Thus, the Hippo pathway plays critical roles in regulating neural progenitor cell number by affecting proliferation, fate choice, and cell survival.

[Keywords: Mst1/2; Lats1/2; chick spinal cord; CNS; neural stem cells; neurogenesis]

Supplemental material is available at http://www.genesdev.org.

Received August 11, 2008; revised version accepted October 2, 2008.

In the developing vertebrate neural tube, neural progenitor cells reside along the ventricle and form a pseudostratified epithelium. With their ability to carry out rounds of cell divisions and to produce progeny of different fates, neural progenitor cells ultimately give rise to the vast numbers and diverse types of neurons and glia that constitute the mature nervous system (Merkle and Alvarez-Buylla 2006). Molecular pathways controlling neural progenitor cell number are not only essential for achieving the proper size and composition of the nervous system, but are also likely to have participated in the expansion of brain size during evolution (Rakic 1995). Dysregulation of these pathways can lead to malformations and/or tumorigenesis in the nervous system (Walsh 1999; Dyer 2004).

The number of neural progenitor cells can be influenced by their proliferation properties (length and rounds of cell cycles), cell fate decisions (to remain as a progenitor or to differentiate), and survival. Recently, the Hippo pathway has been shown to control cell proliferation and

Corresponding authors.

${ }^{3}$ E-MAIL gage@salk.edu; FAX (858) 597-0824.

${ }^{4}$ E-MAIL pfaff@salk.edu; FAX (858) 453-2573.

Article published online ahead of print. Article and publication date are online at http://www.genesdev.org/cgi/doi/10.1101/gad.1726608. survival in Drosophila (Saucedo and Edgar 2007). Its core components consist of two serine/threonine kinases, the Ste-20 family kinase Hippo (Hpo) and the nuclear Dbf2related (NDR) family kinase Warts (Wts), and a transcriptional coactivator named Yorkie (Yki). Hpo phosphorylates and activates Wts, which in turn phosphorylates Yki. Phosphorylated Yki is sequestered in the cytoplasm and is incapable of activating transcription. Inactivation of either kinase or overexpression of Yki leads to the accumulation of unphosphorylated Yki, which activates genes that promote cell proliferation and survival and causes cancerous growth. Because Yki lacks an intrinsic DNA-binding activity, its target gene specificity is dictated by interactions with other factors.

In addition to the core components, adaptor proteins Salvador (Sav) and Mats facilitate the phosphorylation cascade. The cell surface protocadherin Fat and membrane-associated proteins Merlin and Expanded act as upstream activators of the pathway. Most of the identified components of the fly Hippo pathway have conserved vertebrate orthologs, and a number of the Hippo pathway components have been implicated in human cancers (Saucedo and Edgar 2007). Mice lacking lats1, the vertebrate ortholog of wts, develop soft-tissue sarcomas and ovarian tumors and are sensitized to carcinogenic treatments (St John et al. 1999). Overexpression of YAP, the 
vertebrate ortholog of $y k i$, in the mouse liver dramatically increases liver size; likewise, its overexpression in the intestine expands the progenitor pool (Camargo et al. 2007; Dong et al. 2007). These findings suggest that individual components of the Hippo pathway have conserved functions in regulating cell proliferation and survival in vertebrates. However, the functional interactions of these components have not been studied in vivo, in particular with the goal of defining developmental signaling pathways.

A missing key component of the Hippo pathway is the DNA-binding transcription factor(s) that interacts with Yki/YAP and guides it to its target genes. YAP has been shown to interact with nine proteins/protein families in cultured mammalian cells, including Yes, Runx, EBP50, p73, p53BP-2, TEAD, 14-3-3, ErbB-4, and hnRNP U (Saucedo and Edgar 2007), at least five of which are transcription factors/cofactors. The lack of a physiological assay system has made it difficult to determine which one is the cognate partner that mediates YAP function in vivo. Here we show that the vertebrate Hippo pathway regulates neural progenitor cell number during neural tube development and the TEA domain transcription factor (TEAD) is the cognate DNA-binding partner of YAP. During the preparation of this manuscript, works in Drosophila and a mammalian epithelial cell line have also found that the TEAD proteins link YAP/Yki to its target genes (Goulev et al. 2008; Wu et al. 2008; Zhang et al. 2008; Zhao et al. 2008).

\section{Results}

Overexpressing YAP causes a marked expansion of the neural progenitor pool

In the early vertebrate neural tube, progenitor cells and neurons occupy distinct regions along the medial-lateral axis of the neural tube, whereby progenitor cells reside medially along the ventricle and cells undergoing differentiation migrate laterally to form the mantle zone of post-mitotic neurons. In the mouse neural tube, YAP is expressed by ventricular zone progenitor cells (Milewski et al. 2004). To study the function of the Hippo pathway in vertebrate neural development, we first defined the expression pattern of YAP in the chick neural tube. At Hamburger-Hamilton (HH) stage (Hamburger and Hamilton 1992) 27-28 (equivalent to mouse embryonic day 12), YAP mRNA was detected in the ventricular zone (Fig. 1A). In the human YAP protein, the key site phosphorylated by the Hippo kinase cascade is S127 (Dong et al. 2007; Zhao et al. 2007), which corresponds to S126 in chick YAP. An antibody specific for the phosphorylated YAP (pYAP) revealed that, within the neural tube, the domain of pYAP matched the progenitor zone demarcated by the neural progenitor cell marker Sox2 (Fig. $1 \mathrm{~B}, \mathrm{C}$; Bylund et al. 2003). The pYAP labeling pattern suggests that the Hippo signaling pathway is active in neural progenitor cells.

To study the function of this pathway, we electroporated a constitutive YAP expression clone into one side of the chick neural tube at $\mathrm{HH} 12-14$, at which time the neural tube is composed almost entirely of progenitor cells. At $22 \mathrm{~h}$ post-electroporation (hpe), the transfected side exhibited reduced neuronal differentiation, as monitored by the neuronal marker Tuj1 (Fig. 1D). At 45 hpe, in addition to the reduced differentiation (data not shown), there was a marked increase in the number of Sox $2^{+}$progenitor cells. In mild cases, ectopic Sox $2^{+}$cells were found in the mantle zone of the transfected side (Fig. 1E). Most often, the transfected side filled almost entirely with Sox $2^{+}$cells, these cells expanded into the ventricle, accompanied by the deformation of the neural tube (Fig. 1F). The increase in progenitor cell number became even more evident $3 \mathrm{~d}$ post-transfection, with the transfected side being significantly broader than the control side (Fig. 1G). The ectopic Sox $2^{+}$cells in the mantle zone continued to proliferate and were labeled by 5-bromodeoxyuridine (BrdU) (Fig. 1H). In the neural tube, progenitor cells form a pseudostratified epithelium with their apical ends anchored at the ventricular surface via adherens junctions, and their nuclei oscillate across the width of the ventricular zone, where they undergo mitosis at the ventricular surface (Gotz and Huttner 2005). The presence of ectopic phospho-histone $\mathrm{H} 3^{+}$mitotic cells (pH3) (Fig. 1I) that became self-organized around foci of $\mathrm{aPKC}^{+}$adherens junctions (Fig. 1J) indicated that YAP overexpression had induced the formation of rosettes similar to the Homer-Wright rosettes found in many kinds of human neurological tumors (Dyer 2004). These results provide evidence that overactivation of YAP, a critical effector of the Hippo pathway, causes an unchecked expansion of the neural progenitor pool.

A transcriptionally active form of TEAD dramatically increases neural progenitor cell number

Although YAP has been shown to interact with at least five transcription factors/cofactors in cultured cells, including Runx, TEAD, ErbB-4, p73, and hnRNP U, which one mediates YAP function in vivo remains unknown. Five lines of evidence suggest that TEAD may participate in YAP-mediated gene regulation in neural progenitor cells. First, its DNA-binding domain is conserved across all eukaryotes (Anbanandam et al. 2006); thus it potentially could be a component of the evolutionarily conserved Hippo pathway. Second, homozygotes of strong alleles of scalloped $(s d)$, which encodes the Drosophila TEAD protein, die as first instar larvae (Campbell et al. 1992), similar to yki-null mutants (Huang et al. 2005). Third, the four mammalian TEAD proteins, TEAD1-4, are all expressed in the ventricular zone of the neural tube (Milewski et al. 2004). Fourth, mouse TEAD has been shown to activate the promoter of Pax3, a transcription factor expressed by neural progenitor cells (Milewski et al. 2004). Finally, TEAD proteins have been shown to lack intrinsic transactivation activity and require an obligate coactivator to turn on transcription (Vassilev et al. 2001).

At least three TEAD proteins-TEAD1, TEAD3, and TEAD4-are encoded in the chick genome (Azakie et al. 


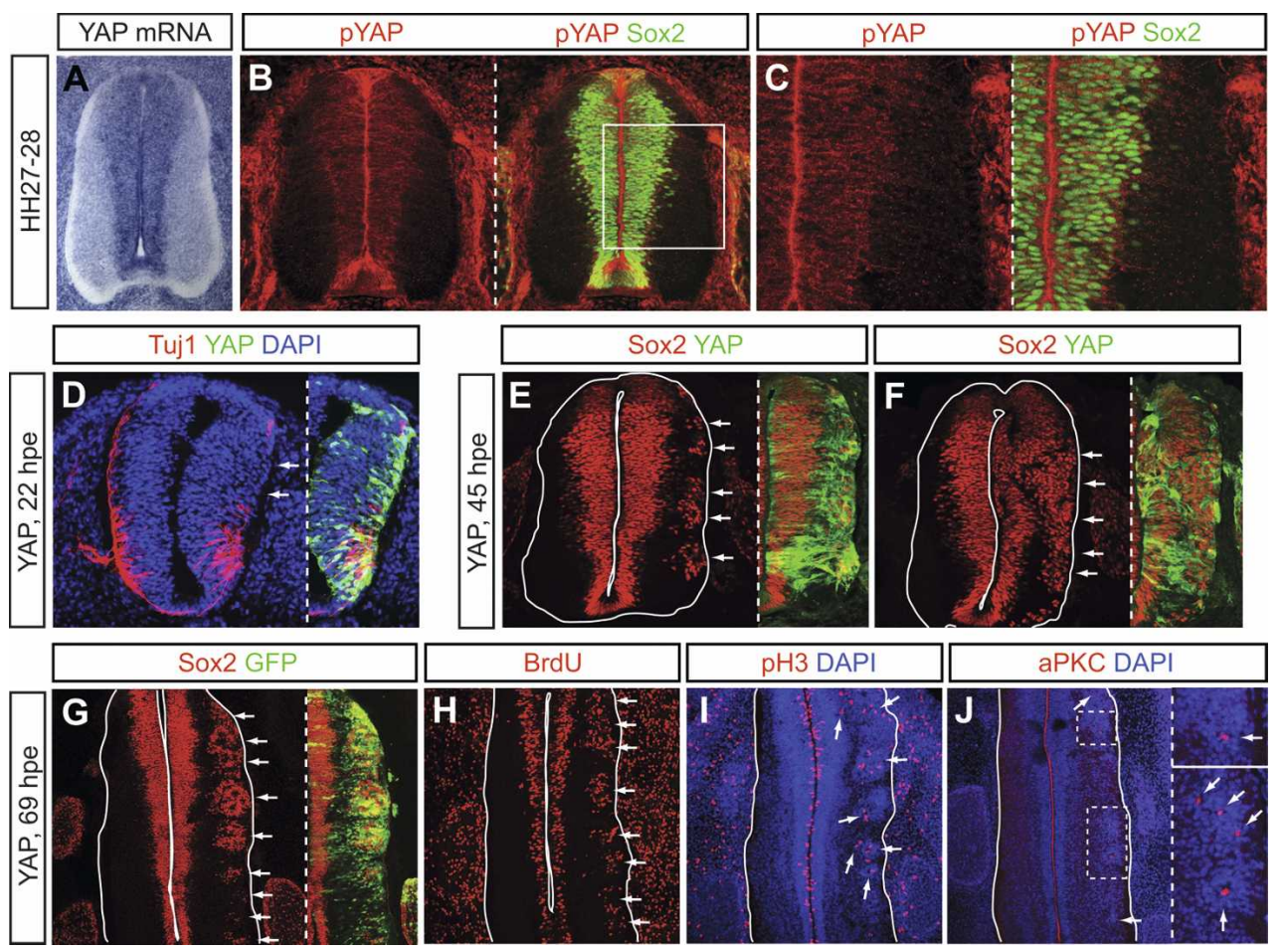

Figure 1. Overexpressing YAP in the neural tube results in reduced differentiation and a marked expansion of the progenitor population. ( $A$ ) In situ hybridization shows YAP expression in the ventricular zone of HH27-28 chick neural tube. (B,C) Phosphorylated YAP (pYAP) was detected in neural progenitor cells marked by Sox 2 immunostaining. The boxed area in $B$ is shown at higher magnification in $C$. $(D-G)$ Transfecting YAP caused a reduction in Tuj1 $1^{+}$neurons $(D$, arrows $)$ and an increase in Sox $2^{+}$progenitor cells $(E-G$, arrows). In all cases, the right side of the neural tube was transfected. Images on the left of the dashed lines show the full neural tube cross sections, those on the right only the transfected side. $(D-F)$ Overexpressed YAP was detected by a YAP antibody, which was not sensitive enough to detect endogenous YAP. $(G)$ At 69 hpe, the transfected side was marked by cotransfected GFP. (H-J) Ectopic progenitor cells continued to proliferate at 69 hpe (shown in $H$ ). They divided at the mantle zone (I, arrows) and formed tumor-like rosettes with foci of $\mathrm{PPKC}^{+}$adherens junctions $(J$, arrows; areas in dashed squares are shown at higher magnification in the images to the right of the dashed line).

1996). With an antibody specific for TEAD1 (Supplemental Fig. S1A), we examined its expression pattern in the HH27-28 neural tube. At cervical, brachial, and lumbar levels, TEAD1 was expressed in the ventricular zone but not in the Tuj $1^{+}$mantle zone (Fig. 2A). Closer examination revealed that the cell domain expressing TEAD1 was slightly broader than the progenitor zone marked by Sox2 and pYAP (Fig. 2B; Supplemental Fig. S1B). Some $\mathrm{TEAD}^{+}$cells also expressed the proneural basic-helixloop-helix (bHLH) transcription factor Neurogenin 2 (Ngn2) (Fig. 2B; Ma et al. 1996). At the thoracic level, TEAD1 was also present in a group of cells located at the ventral-lateral region of the neural tube (Supplemental Fig. S1C-E). We also detected TEAD4 mRNA in the ventricular zone of the chick neural tube (Supplemental Fig. $\mathrm{S} 1 \mathrm{~F})$. Unfortunately, several in situ hybridization probes we tested for TEAD3 did not yield specific signals, and therefore we cannot yet conclude whether TEAD3 is expressed in the chick neural tube. We confirmed the interaction between YAP and TEAD in neural progenitor cells by performing coimmunoprecipitation in cell lysates of HH27-28 forebrain and midbrain, where both YAP and TEAD1 are expressed in the ventricular progenitor zone (Supplemental Fig. S1G; data not shown).
As a first step in testing the hypothesis that TEAD is the cognate DNA-binding factor that mediates YAP function in the neural tube, we examined whether increasing TEAD levels could mimic the effect of YAP overexpression. We did not detect a change in neural progenitor proliferation or differentiation following TEAD1 overexpression (Fig. 2C,D,M). This result is consistent with TEAD proteins lacking intrinsic transactivation activity. It also implies that the amount/availability of the coactivator is limited in neural progenitor cells.

If TEAD mediates the function of YAP, expressing a transcriptionally active form of TEAD should recapitulate the phenotype of YAP overexpression. We engineered such a TEAD1 construct by replacing the YAPbinding domain (Vassilev et al. 2001) with the activation domain (AD) of VP16 (TEA-VP16) (Fig. 2E), as previous studies in cultured cells have shown that the activity of YAP AD is as potent as that of VP16 AD (Yagi et al. 1999). Structural studies suggest that the guanidino group of R59 (the 59th amino acid of the TEA domain) is likely to contact DNA directly (Anbanandam et al. 2006). We therefore generated a DNA binding-defective control by mutating R59 into lysine (TEA $\left.{ }^{\mathrm{R} 59 \mathrm{~K}}-\mathrm{VP} 16\right)$ (Fig. 2E). An allele of Drosophila sd harboring the same 


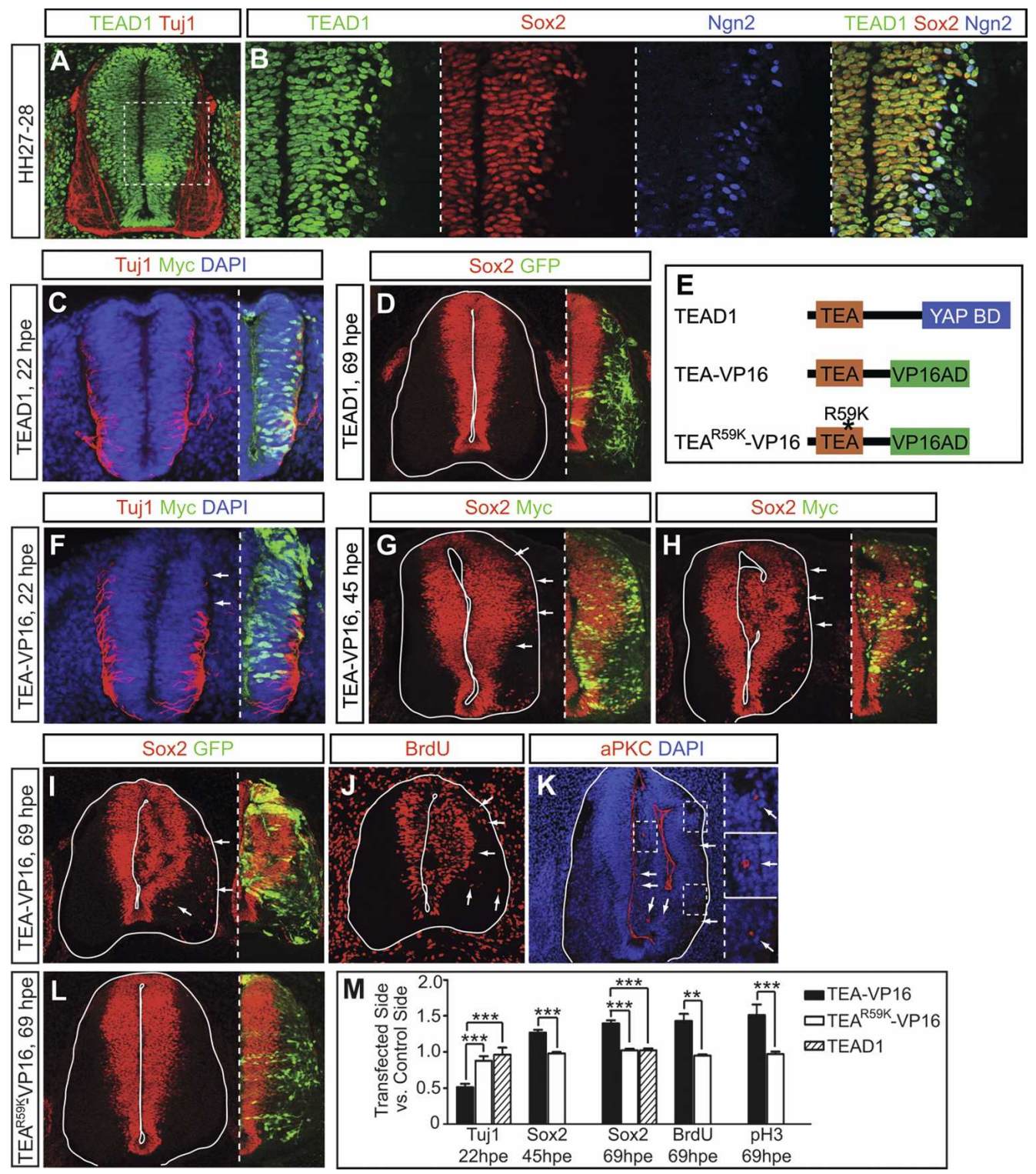

Figure 2. Overexpressing a transcriptionally active form of TEAD1 increases neural progenitor cell number. $(A, B)$ TEAD1 expression pattern examined by immunostaining. The region examined in $B$ corresponds to that within the dashed square in $A$. $(C, D)$ Overexpressing TEAD1 did not significantly affect differentiation $(C)$ or progenitor cell number $(D)$. $(E)$ Domain structures of TEAD1 and derivatives. Mammalian and avian TEAD proteins are composed of an N-terminal TEA domain and a C-terminal YAP-binding domain (YAP BD) connected by a linker region. (VP16AD) The activation domain of VP16. (F-K) Reduced neuronal differentiation $(F$, arrows) and increased progenitor cells ( $G-I$, arrows) upon TEA-VP16 expression. Transfected side showed severe dysplasia with disruptions of ventricular surface and the formation of tumor-like rosettes $(K$, arrows; areas in dashed squares are shown at higher magnification in the images right of the dashed line). (L) TEA ${ }^{\mathrm{R} 59 \mathrm{~K}}-\mathrm{VP} 16$ did not have a strong effect on progenitor cell number. $(C, F-H)$ TEAD constructs have an N-terminal Myc tag and were detected by a Myc antibody. $(D, I, L)$ At 69 hpe, the transfected side was marked by cotransfected GFP. $(M)$ Quantifications comparing the transfected side with the control side. In this and all following graphs, results are mean \pm SEM. $\left.{ }^{\star}\right) P<0.05 ;\left(^{\star \star}\right) P<0.01 ;\left(^{\star \star \star}\right) P<0.001$.

mutation is a strong loss-of-function allele (Srivastava et al. 2004). Introducing TEA-VP16 into the neural tube caused phenotypes similar to YAP overexpression: Neuronal differentiation was reduced at 22 hpe (Fig. 2F,M); there was a marked expansion in progenitor cells at 45 hpe, often accompanied by the deformation of the neural tube (Fig. 2G,H,M); and the overproliferation phenotype became even more severe at 69 hpe with the formation of tumor-like rosettes (Fig. 2I-K,M). In contrast, TEA ${ }^{\mathrm{R} 59 \mathrm{~K}}$ VP16 did not significantly alter progenitor cell number (Fig. 2L,M), suggesting that the effect caused by TEAVP16 is dependent on DNA binding. Despite the structural distortion of YAP- and TEA-VP16-transfected neural tubes, dorsal-ventral patterning, determined by the expression of regional markers Pax3, Pax7, and Pax6, was largely normal (data not shown). 


\section{Misexpressing YAP $P^{\Delta 60-89}$ fails to expand the neural progenitor pool}

The above results showed that constitutive expression of YAP and the active form of TEAD caused identical phenotypes. To test whether YAP exerts its effect through TEAD, we generated a YAP mutant by removing 30 amino acids within the TEAD-binding domain $\left(\mathrm{YAP}^{\Delta 60-89}\right.$ ) (Fig. $3 \mathrm{~A}$; Vassilev et al. 2001). This deletion is unlikely to perturb the interactions between YAP and other known transcription partners, which are mediated by either the WW domain or the N terminus of YAP (Yagi et al. 1999; Strano et al. 2001; Komuro et al. 2003; Howell et al. 2004). A coimmunoprecipitation experiment confirmed that this mutant no longer interacted with TEAD1 (Fig. 3B). In contrast to the severe phenotype caused by YAP overexpression, neural tubes transfected with $\mathrm{YAP}^{\Delta 60-89}$ were largely normal (Fig. 3C,D). This result supports the hypothesis that YAP functions through binding to TEAD, although we cannot rule out that the deletion may abolish the interaction between YAP and other unidentified partners.

The increase in progenitor cell number results from accelerated proliferation and reduced cell cycle exit

We next analyzed the mechanisms responsible for the large expansion of neural progenitors associated with

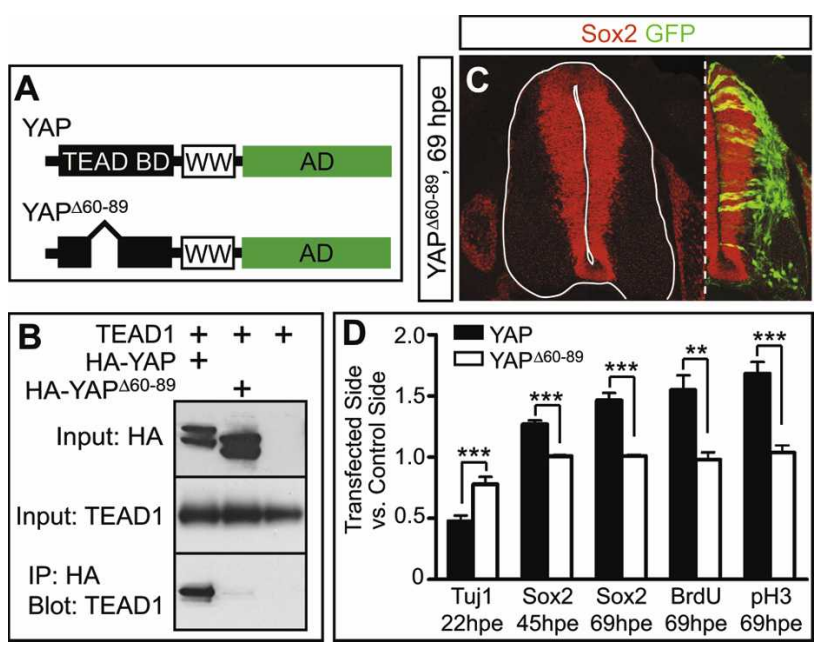

Figure 3. $\mathrm{YAP}^{\Delta 60-89}$ cannot interact with TEAD and fails to increase neural progenitor cell number. $(\boldsymbol{A})$ Domain structures of YAP and the deletion construct. Chick YAP is composed of an N-terminal TEAD-binding domain (TEAD BD), a central WW domain, and a C-terminal activation domain (AD). (B) A coimmunoprecipitation experiment shows that $\mathrm{YAP}^{\Delta 60-89}$ could not interact with TEAD1. 293T cells were transfected with TEAD1 and HA-YAP or HA-YAP ${ }^{\Delta 60-89}$. Cell lysates were immunoprecipitated (IP) with an HA antibody and blotted with a TEAD1 antibody. (C) Overexpressing YAP ${ }^{\Delta 60-89}$ did not significantly increase neural progenitor cells. The transfected side was marked by cotransfected GFP. (D) Quantifications comparing the effects of misexpressing YAP and $\mathrm{YAP}^{\Delta 60-89}$.
YAP and TEA-VP16 misexpression. An increase in cell number can result from accelerated proliferation, decreased cell cycle exit, and/or reduced cell death. The level of cell death in the early neural tube is very low (Kuida et al. 1998). Thus, reducing cell death is unlikely to have contributed significantly to the large increase in cell number. The reduction in neuronal differentiation upon YAP and TEA-VP16 overexpression suggests that cell cycle exit was decreased. At early stages of neural tube development, most cell divisions produce two progenitor cells (Morin et al. 2007). Thus, an increase in the rate of proliferative divisions can exponentially increase cell number. We assessed the cell cycle length of transfected progenitor cells by 30-min BrdU pulselabeling: An increase in the percent of cycling cells that are labeled with BrdU indicates the cell cycle length has shortened. We quantified the fraction of $\mathrm{BrdU}^{+}$ cells among the transfected cells that remained cycling at the time of labeling ( $22 \mathrm{hpe}$ ) and compared this fraction with that of the control side to obtain a BrdU-labeling ratio. We used Sox 2 as a marker for cycling cells because all Sox2 ${ }^{+}$cells express the proliferating cell marker PCNA at this stage (data not shown). Thus, the BrdU labeling ratio for YAP-transfected neural tubes is $\left(\mathrm{BrdU}^{+} \mathrm{YAP}^{+} \text {Sox } 2^{+} / \mathrm{YAP}^{+} \text {Sox } 2^{+}\right)_{\text {transfected }}$ side $/\left(\mathrm{BrdU}^{+} \mathrm{Sox}^{+} /\right.$ Sox $2^{+}$control side and that for TEA-VP16-transfected ones is $\left(\mathrm{BrdU}^{+} \mathrm{Myc}^{+} \mathrm{Sox}^{+} / \mathrm{Myc}^{+} \mathrm{Sox}_{2}{ }^{+}\right)_{\text {transfected side }} /\left(\mathrm{BrdU}^{+} \mathrm{Sox}^{+} /\right.$ Sox $\left.2^{+}\right)_{\text {control side. }}$ Both YAP- and TEA-VP16-transfected neural tubes had a BrdU-labeling ratio of more than 1 $(1.26 \pm 0.07, n=18$ for YAP; $1.29 \pm 0.06, n=14$ for TEAVP16) (Fig. 4A), suggesting that the transfected cells have a shortened cell cycle length. In comparison, the cell cycle length of $\mathrm{YAP}^{\Delta 60-89}$ - and TEA ${ }^{\mathrm{R} 59 \mathrm{~K}}$-VP16-transfected cells was not significantly different from that of untransfected cells (BrdU labeling ratio was $1.03 \pm 0.06$, $n=15$ for $\mathrm{YAP}^{\Delta 60-89}$ and $0.98 \pm 0.07, n=11$ for TEA $^{\mathrm{R} 59 \mathrm{~K}}$. VP16). Together, these results suggest that the increase in neural progenitor cell number was caused by accelerated cell cycle progression and reduced cell cycle exit.

\section{Overexpressing YAP and TEA-VP16 induces Cyclin D1 expression}

We next searched for the molecular mechanism underlying the changes in cell cycle parameters. Hyperactivating YAP has been shown to induce cyclin D1 and $c-M y c$ expression in the intestine and liver, respectively $(\mathrm{Ca}-$ margo et al. 2007; Dong et al. 2007). We therefore surveyed a number of genes that have been implicated in cell cycle control, including cyclin D1, cyclin D2, c-Myc, and $N$-Myc. Among them, cyclin D1 mRNA was strongly up-regulated upon YAP and TEA-VP16 overexpression (Fig. 4B,C; cf. Supplemental Fig. S2).

D-type cyclins (D1, D2, and D3), in response to mitogenic signals, act during early to mid-G1 phase to drive cell cycle progression (Massague 2004). We asked whether cyclin D1 up-regulation was responsible for the phenotypes associated with YAP and TEA-VP16 overexpression. In cultured cells, overexpressing cyclin D1 has been shown to shorten cell cycle length (Quelle et al. 1993). 

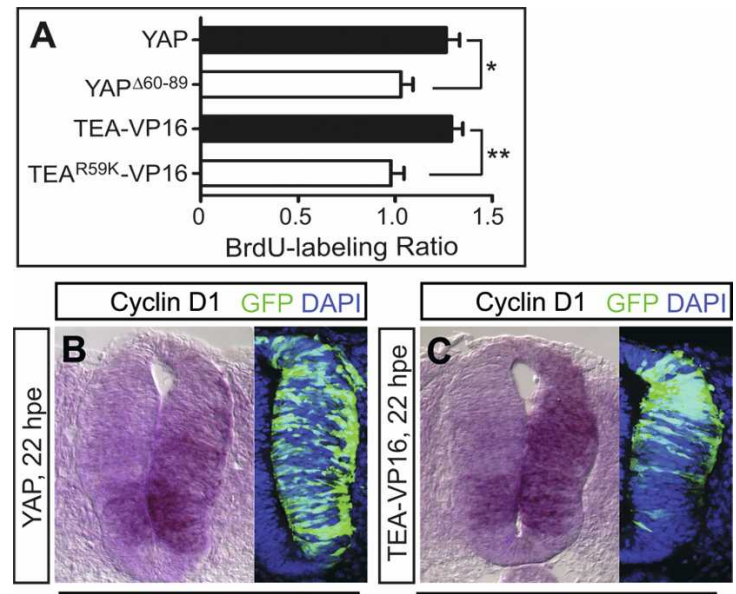

Tuj1 GFP DAPI
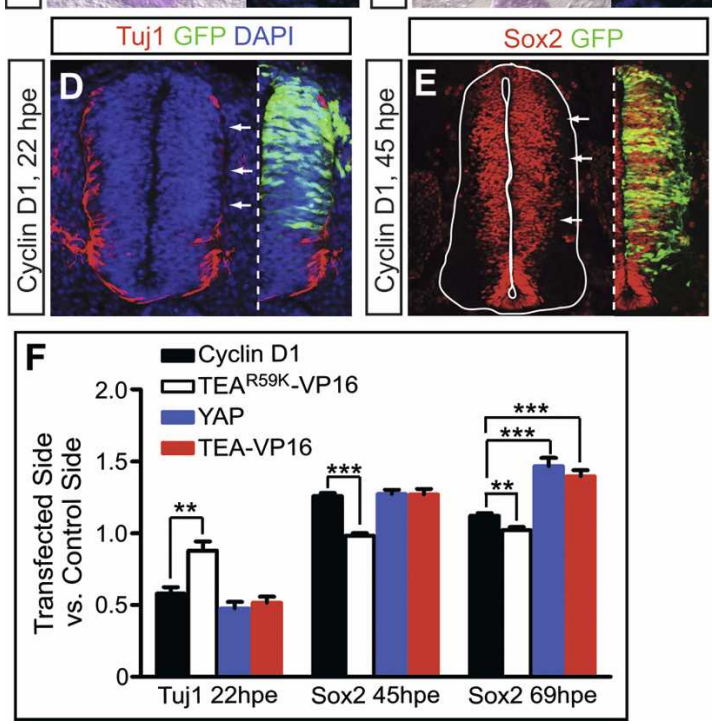

Figure 4. YAP and TEA-VP16 overexpression promotes cell cycle progression. $(A)$ Overexpressing YAP and TEA-VP16 accelerated the cell cycle. $(B, C)$ In situ hybridization (ISH) reveals up-regulation of cyclin D1 by YAP and TEA-VP16 transfection. YAP and TEA-VP16 cDNAs are followed by IRES-GFP to monitor their expression. The image next to the ISH photograph shows GFP expression in an adjacent section. $(D, E)$ Overexpressing cyclin D1 reduced differentiation $(D$, arrows) and increased progenitor cell number ( $E$, arrows). Cyclin D1 cDNA is followed by IRES-GFP to monitor its expression. $(F)$ Quantifications comparing the effect of cyclin D1 overexpression with that of YAP and TEA-VP16. Neural tubes transfected with $\mathrm{TEA}^{\mathrm{R} 59 \mathrm{~K}-\mathrm{VP} 16}$ serve as the baseline.

Thus, cyclin D1 up-regulation likely accounts for the accelerated cell cycle rate in YAP- and TEA-VP16-transfected neural tubes. Consistent with a previous report (Lobjois et al. 2004), we found that forced expression of cyclin D1 inhibited neuronal differentiation at 22 hpe (Fig. 4D,F). At 45 hpe, there was a substantial increase in the number of Sox $2^{+}$cells in the transfected side $(26 \pm 2 \%$, $n=10$ ) (Fig. 4E,F). The scale of the increase was comparable with that caused by the overexpression of YAP $(27 \pm 3 \%$, $n=10)$ and TEA-VP16 $(27 \pm 4 \%, n=10)$. The morphological effect of cyclin D1 overexpression, however, was quite different from that of YAP and TEA-VP16. Al- though we always observed expansion in the width of the ventricular zone (Fig. 4E, arrows), we found fewer ectopic Sox $2^{+}$cells in the mantle zone and never encountered deformations of the neural tube like those caused by YAP and TEA-VP16 overexpression. By 69 hpe, the increase in Sox $2^{+}$cells, though still present, was much diminished $(12 \pm 2 \%, n=6)$ and was significantly lower than that caused by YAP and TEA-VP16 transfection (Fig. 4F). Together, these data suggest that cyclin D1 upregulation could partially account for the overproliferation phenotype caused by YAP and TEA-VP16 overexpression.

\section{YAP and TEAD loss of function leads to increased cell} death

To study the effect of YAP loss of function in the neural tube, we generated two shRNA plasmids. Both shRNAs inhibited the expression of cotransfected YAP cDNA in chick fibroblasts (Supplemental Fig. S3A). Upon introduction into the neural tube, they reduced the level of endogenous YAP mRNA (Fig. 5A,D). The transfected side was much thinner than the control side at 45 hpe, suggestive of increased cell death or/and reduced proliferation. TUNEL assays revealed that both shRNAs caused a marked increase in cell death at 22 hpe (Fig. $5 \mathrm{~B}, \mathrm{E}, \mathrm{F})$. Two observations suggest this phenotype is specific. First, cotransfection of mouse YAP cDNA (mYAP), which is insensitive to the chick YAP shRNAs, completely rescued the cell death phenotype (Fig. 5C,F). Second, a control shRNA consisting of the scrambled sequence of shYAP\#1 did not cause significant cell death (Fig. 5F).

To confirm these findings, we employed an independent method to inhibit YAP function by expressing a dominant-negative construct. We first created a truncation mutant $\left(\mathrm{YAP}^{279}\right)$ in which the activation domain of YAP was removed. We reasoned that, upon misexpression, $\mathrm{YAP}^{279}$ should compete with endogenous YAP for binding to its partners but lack the ability to activate transcription, thereby inhibiting the function of endogenous YAP. We transfected YAP ${ }^{279}$ into the neural tube but did not detect increased cell death or any other effects (data not shown). When we checked the subcellular localization of $\mathrm{YAP}^{279}$, we found that it was almost exclusively cytoplasmic (data not shown). Thus it is possible that the amount of nuclear YAP $^{279}$ was insufficient to compete with endogenous YAP. We therefore attached a nuclear localization signal (NLS) to $\mathrm{YAP}^{279}\left(\mathrm{YAP}_{\mathrm{N}}{ }^{279}\right)$ (Fig. 5G). Consistent with the cell death phenotype triggered by shRNA-mediated knockdown, misexpressing $\mathrm{YAP}_{\mathrm{N}}{ }^{279}$ caused a fivefold increase in cell death (Fig. $5 \mathrm{H}, \mathrm{J})$. In addition to the increased TUNEL signals, cleaved caspase 3 was strongly induced by $\mathrm{YAP}_{\mathrm{N}}{ }^{279}$ (Supplemental Fig. $\mathrm{S} 3 \mathrm{~B})$. A deletion within $\mathrm{YAP}_{\mathrm{N}}{ }^{279}$ that abolishes the interaction between YAP and TEAD $\left(\mathrm{YAP}_{\mathrm{N}}{ }^{279 \Delta}\right)$ (Fig. $\left.5 \mathrm{G}\right)$ greatly reduced the apoptotic effect (Fig. 5I,J), suggesting that the dominant-negative activity of $\mathrm{YAP}_{\mathrm{N}}{ }^{279}$ was probably achieved by competing with endogenous YAP for TEAD binding. 

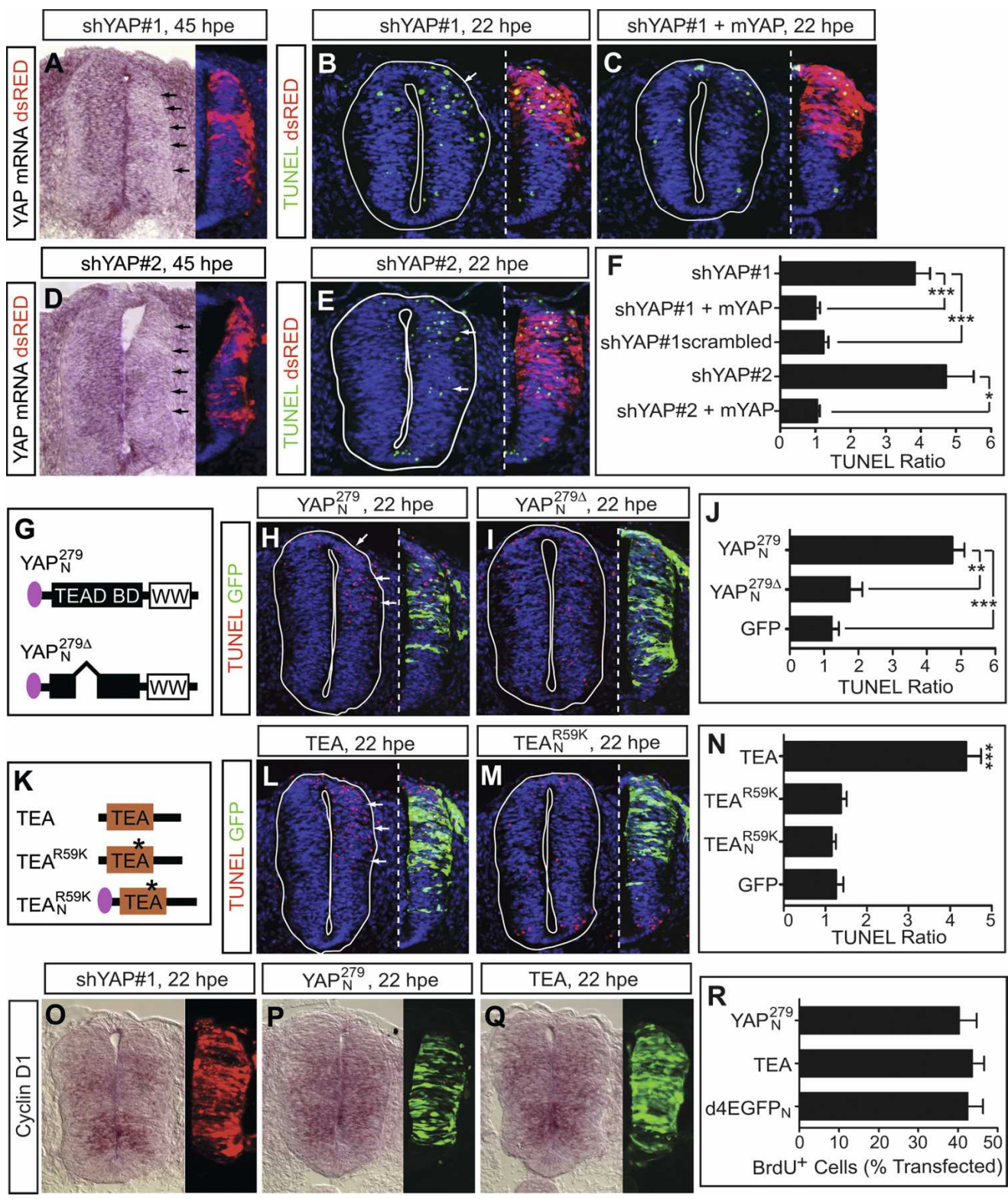

Figure 5. YAP and TEAD loss of function in the neural tube leads to increased cell death. $(A)$ ISH shows reduced YAP mRNA level in shYAP\#1-transfected neural tube (arrows). The shRNA vector also encodes dsRED to mark transfected cells. The image next to the ISH photograph shows dsRED expression in an adjacent section. Blue signals are DAPI. $(B, C)$ TUNEL assay detected increased cell death in shYAP\#1-transfected side $(B$, arrows). The cell death phenotype was rescued by cotransfecting mYAP $(C)$. $(D, E)$ shYAP\#2 reduced YAP mRNA level ( $D$, arrows) and caused increased cell death (E, arrows). ( $F$ ) Quantifications of the cell death phenotype. For each embryo, the sum of TUNEL signals in the transfected side (four to 10 sections per embryo) was divided by that in the control side to obtain a TUNEL ratio. $(G)$ Domain structures of YAP dominant-negative constructs. The pink oval represents $\mathrm{NLS}(H-J) \mathrm{YAP}_{\mathrm{N}}{ }^{279}$, but not $\operatorname{YAP}_{\mathbf{N}}{ }^{279 \Delta}$, caused increased cell death $(H$, arrows). The transfected side was marked by cotransfected GFP. (K) Domain structures of TEAD dominant-negative constructs. $(L-N)$ TEA, but not $\mathrm{TEA}^{\mathrm{R} 59 \mathrm{~K}}$ or $\mathrm{TEA}_{\mathrm{N}}{ }^{\mathrm{R} 59 \mathrm{~K}}$, triggered increased cell death $($ arrows in $L$ ). The transfected side was marked by cotransfected GFP. (O-Q) ISH shows inhibiting YAP and TEAD did not significantly affect cyclin D1 expression. Transfection efficiency was monitored by the expression of dsRED encoded in the shRNA vector $(O)$ or cotransfected GFP $(P, Q)$ in adjacent sections. $(R)$ Inhibiting YAP and TEAD with YAP $_{\mathrm{N}}{ }^{279}$ and TEA, respectively, did not significantly affect BrdU incorporation during a 2-h labeling period. Transfected embryos were harvested at 22 hpe. Neural tubes transfected with $\mathrm{d}_{4} \mathrm{EGFP}_{\mathrm{N}}$ were used as the control.

Due to the presence of multiple TEAD proteins in the neural tube and the difficulty we encountered when trying to knock them down simultaneously with shRNAs, we resorted to the dominant-negative approach to study the loss-of-function effect of TEAD proteins. The dominant inhibitor, TEA, contains only the TEA domain and surrounding sequences of TEAD1 but lacks the YAP-binding domain (Fig. 5K). It should compete with endogenous 
TEAD proteins for DNA binding but lack the ability to activate transcription. For the negative control, we introduced the R59K mutation that abolishes/weakens the DNA-binding ability of TEA domain (TEA ${ }^{\mathrm{R} 59 \mathrm{~K}}$ ) (Fig. 5K). Upon examining the subcellular localization of these proteins in transfected neural tubes, we found that, while TEA was enriched in the nucleus, TEA $^{\text {R59K }}$ was present in both the nucleus and the cytoplasm (data not shown). Therefore, we attached a NLS to its $\mathrm{N}$ terminus (TEA ${ }_{N}{ }^{\text {R9K }}$ ) (Fig. 5K). Overexpression of TEA, but not $\mathrm{TEA}^{\mathrm{R} 59 \mathrm{~K}}$ or $\mathrm{TEA}_{\mathrm{N}}{ }^{\mathrm{R} 5 \mathrm{~K}}$, caused a marked increase in apoptosis detected by TUNEL assays (Fig. 5L-N) and immunostaining for cleaved caspase 3 (Supplemental Fig. S3C).

We next investigated whether YAP and TEAD loss of function affected proliferation. Since YAP and TEA-VP16 overexpression strongly elevated cyclin D1 mRNA levels, we examined whether their loss of function diminished cyclin D1 expression. To our surprise, we did not detect a decrease in cyclin D1 mRNA in neural tubes transfected with YAP shRNAs, YAP inhibitor $\mathrm{YAP}_{\mathrm{N}}{ }^{279}$, or TEAD inhibitor TEA at 8, 12, 16, and 22 hpe (Fig. 5O-Q; Supplemental Fig. S3F-I; data not shown). We also measured proliferation by BrdU labeling. The fraction of $\mathrm{YAP}_{\mathrm{N}}{ }^{279}$ - and TEA-transfected cells that incorporated BrdU was the same as that of cells transfected with the control plasmid d4EGFP $_{\mathrm{N}}$ (nuclear-localized destabilized EGFP) (Fig. 5R; Supplemental Fig. S3D,E). We also evaluated neuronal differentiation with markers including Tuj1, neurofilament-associated protein $(\mathrm{NF})$, and Lim $1 / 2$ and did not observe obvious phenotypes in neural tubes transfected with YAP shRNAs, YAP $_{\mathrm{N}}{ }^{279}$, or TEA except for a small decrease in the number of cells expressing these markers (data not shown), which was probably due to the increased cell death. Dorsal-ventral patterning of transfected neural tubes also appeared normal (data not shown). Together, these findings suggest that cell cycle progression and cell fate specification can operate in the absence of YAP and TEAD function.

Repressing YAP and TEAD target genes induces cell cycle exit and neuronal differentiation

Next, we investigated the effect of repressing YAP and TEAD downstream genes. We replaced the activation domain of YAP and the YAP-binding domain of TEAD1 with the transcription repression domain of Drosophila Engrailed (YAP ${ }^{279}$-EnR and TEA-EnR; Fig. 6A). Consistent with YAP and TEAD loss-of-function phenotypes, introducing each construct into the neural tube induced cell death at 22 hpe (data not shown). Strikingly, in contrast to YAP loss of function, which had no obvious effect on proliferation, most cells transfected with YAP ${ }^{279}$. EnR exited the cell cycle by 22 hpe, as shown by the lack of BrdU incorporation (Fig. 6B,H). Most transfected cells had migrated to the mantle zone and were expressing neuronal markers NF and Lim $1 / 2$ (Fig. 6B-D,H). There was an increase in the number of neurons in the transfected side compared with the control side (Fig. 6H, right graph), suggesting that $\mathrm{YAP}^{279}$-EnR promoted neuronal differentiation. These phenotypes were absent in neural tubes transfected with the deleted version, $\mathrm{YAP}^{279 \Delta}$-EnR (Fig. 6H; Supplemental Fig. S4A-D). Misexpressing TEAEnR, but not TEA ${ }^{\text {R59K}}$-EnR, also triggered cell cycle exit and neuronal differentiation, and the differentiation phenotype was even stronger (Fig. 6E-H; cf. Supplemental Fig. S4E-G).

Next we examined the earlier events that led to neuronal differentiation. As early as 8 hpe, both $\mathrm{YAP}^{279}$-EnR and TEA-EnR had repressed cyclin D1 expression (Fig. $6 \mathrm{I}, \mathrm{L})$. Although we did not observe a reduction in the mRNA levels of Hes1 and Hes5-bHLH factors that inhibit neuronal differentiation and maintain the progenitor population (Hatakeyama et al. 2004) —or an upregulation of the proneural gene Ngn2 (data not shown), the expression of the neurogenic bHLH factor NeuroM/ NeuroD4/Math3 (Roztocil et al. 1997; Lee and Pfaff 2003) was strongly induced by TEA-EnR at 8 hpe (Fig. $6 \mathrm{M})$. Induction of NeuroM by $\mathrm{YAP}^{279}$-EnR was weak at 8 hpe (data not shown) but became much stronger at 12 hpe (Fig. 6J). The linking of neuronal differentiation to NeuroM regulation prompted us to check whether NeuroM expression was affected in the opposite manner in YAPand TEA-VP16-transfected neural tubes, in which differentiation was inhibited. Indeed, both YAP and TEAVP16 overexpression reduced NeuroM mRNA levels (Fig. 6K,N) without inducing Hes1 or Hes5 expression (data not shown). Together, these data demonstrate that repressing YAP and TEAD target genes leads to cell cycle exit and neuronal differentiation. These effects are, at least partially, due to the down-regulation of cyclin D1 and the induction of NeuroM.

\section{Epistasis analyses of YAP and TEAD}

Our manipulations of YAP and TEAD functions, either through gain-of-function approaches, loss-of-function approaches, or by repressing their target genes, yielded similar results for these two proteins, strongly suggesting that YAP and TEAD act in the same pathway. The deletion mutants of YAP $(\Delta 60-89)$ that cannot interact with TEAD were inactive in all three settings, indicating that YAP operates through binding to TEAD. To further confirm that TEAD is the DNA-binding partner of YAP in the neural tube, we performed double-transfection experiments to test their epistatic relationship.

If YAP functions by acting as the coactivator of TEAD, then the active form of TEAD, TEA-VP16, should be able to rescue YAP loss of function. Indeed, cotransfection of TEA-VP16 with YAP shRNA largely blocked the cell death phenotype (Fig. 7A,B). Conversely, when YAP was cotransfected with TEA, the dominant inhibitor of TEAD proteins, it was unable to significantly reduce the apoptosis triggered by TEAD loss of function (Fig. 7C,D). Moreover, whereas YAP overexpression inhibited differentiation, cotransfection of TEA-EnR completely reversed this effect, resulting in most transfected cells expressing neuronal markers (Fig. 7E,G), a phenocopy of the TEAEnR single transfection (Fig. 6F-H). Likewise, cotrans- 
Figure 6. Repressing YAP and TEAD downstream genes leads to cell cycle exit and neuronal differentiation. (A) Domain structures of $\mathrm{YAP}^{279}$-EnR and TEA-EnR. EnR: the repression domain of Engrailed. $(B-H)$ Repressing YAP and TEAD target genes through $\mathrm{YAP}^{279}$-EnR and TEA-EnR, respectively, promoted cell cycle exit $(B, E, H)$ and neuronal differentiation $(C, D, F, G$, arrows, $H)$. YAP ${ }^{279}$-EnR was detected by a YAP antibody. TEA-EnR has a Myc tag and was detected by a Myc antibody. $(I-N)$ ISH shows that $\mathrm{YAP}^{279}$-EnR and TEA-EnR repressed $c y$ clin D1 (I,L, arrows) and induced NeuroM $(J, M$, arrows), whereas overexpressing YAP and TEA-VP16 reduced NeuroM mRNA levels ( $K, N$, arrows). Transfection efficiency was monitored by GFP expression-either from the cotransfected GFP-expressing plasmids $(I, J, L, M)$ or the downstream IRES-GFP $(K, N)$-in adjacent sections.
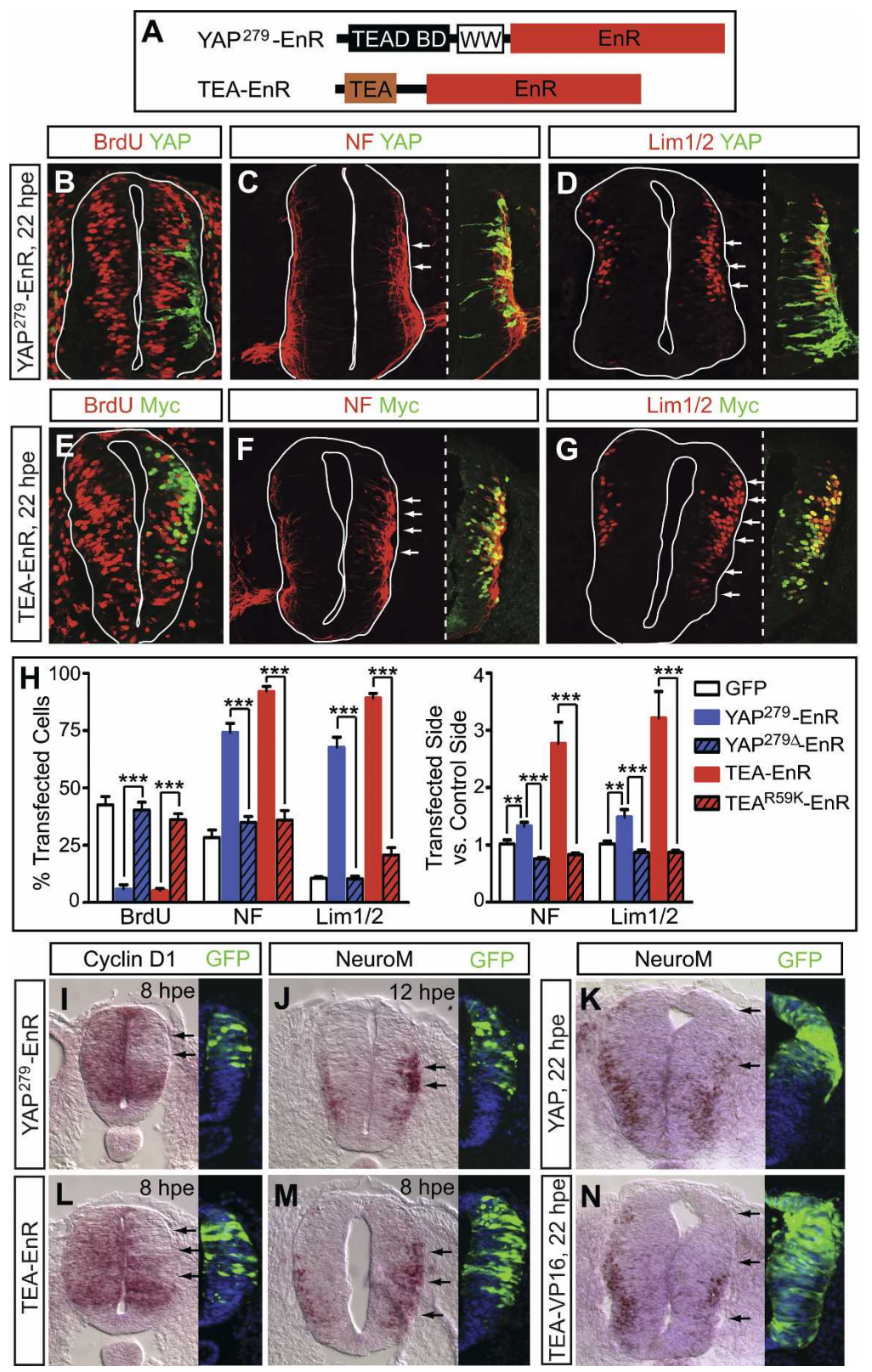

fection of YAP ${ }^{279}$-EnR and TEA-VP16 led to reduced differentiation (Fig. 7F,G), similar to the effect of TEAVP16 overexpression and opposite to that of YAP ${ }^{279}$. EnR. These experiments establish that TEAD is epistatic to YAP and YAP functions through TEAD.

The upstream kinases of the Hippo pathway regulate neural progenitor proliferation and survival

Finally, we investigated whether the upstream kinases of the Hippo pathway regulate neural progenitor cell number. The vertebrate orthologs of $h p o, M s t 1$ and $M s t 2$, and those of wts, Lats1 and Lats2, are all widely expressed (Creasy and Chernoff 1995a,b; Tao et al. 1999; McPherson et al. 2004). Studies using cultured cells or recombi- nant proteins have confirmed that the Hpo-Wts-Yki phosphorylation cascade is conserved for the vertebrate orthologs (Chan et al. 2005; Dong et al. 2007; Zhao et al. 2007). However, the functions of Mst $1 / 2$ and Lats $1 / 2$ in neural development are largely unexplored.

To inhibit Mst1/2 function, we generated a kinasedead version of Mst2 (Mst2 $\left.{ }^{\mathrm{KD}}\right)$, which has been shown to have a dominant-negative effect on the endogenous kinase (Wu et al. 2003). Transfecting Mst $2^{\mathrm{KD}}$ into the neural tube induced cyclin $D 1$ expression at 22 hpe (Fig. $8 \mathrm{~A})$. At $69 \mathrm{hpe}$, the ventricular zone of the transfected side became noticeably wider than the control side, with clusters of ectopic Sox $2^{+}$cells in the mantle zone (Fig. $8 B)$. The number of Sox $2^{+}$cells increased $14 \pm 1 \%$ $(n=15)$ compared with the untransfected side (Fig. 8E). 


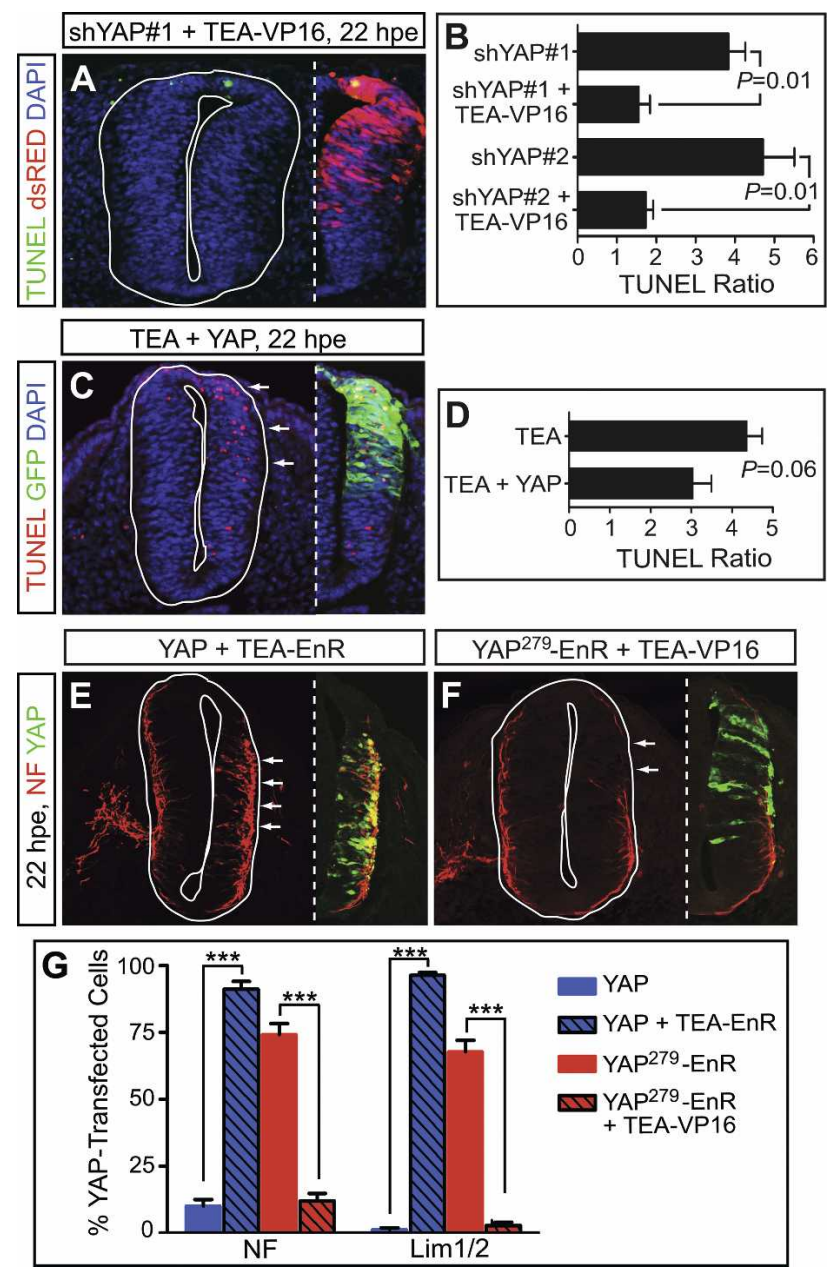

Figure 7. Epistasis analyses of YAP and TEAD. $(A, B)$ TUNEL assays show that cotransfecting TEA-VP16 with YAP shRNAs rescued the cell death phenotype. Transfected cells were marked by dsRED expressed from the shRNA vector. TUNEL ratios are shYAP\#1: $3.8 \pm 0.4, n=12$; shYAP\#1 + TEA-VP16: $1.6 \pm 0.3, n=4$; shYAP\#2: $4.7 \pm 0.8, n=9$; shYAP\#2 + TEAVP16: $1.7 \pm 0.2, n=6$. $(C, D)$ Cotransfecting YAP with TEA could not rescue the cell death phenotype (arrows in C). YAP cDNA is followed by IRES-GFP to mark transfected cells. TUNEL ratios are TEA: $4.4 \pm 0.4, n=6$; TEA + YAP: $3.1 \pm 0.4$, $n=11 .(E-G)$ Cotransfecting TEA-EnR with YAP increased neuronal differentiation (E, arrows), whereas cotransfecting TEAVP16 with YAP ${ }^{279}$-EnR reduced it (F, arrows). In both cases, the phenotype resembled that of the single transfection of the TEAD variant and opposite to that of the YAP variant. A YAP antibody was used to label YAP- and YAP ${ }^{279}$-EnR-transfected cells.

We also engineered a kinase-dead mutant of Lats2 (Lats $2^{\mathrm{KD}}$; Chan et al. 2005) as well as an shRNA targeting Lats1 (shLats1) (Supplemental Fig. S5). Cotransfection of shLats 1 and Lats $2^{\mathrm{KD}}$, similar to Mst $2^{\mathrm{KD}}$ misexpression, increased cyclin D1 mRNA levels and the number of Sox $2^{+}$cells (Fig. $8 \mathrm{C}-\mathrm{E}$ ). These results are consistent with the model that endogenous Mst1/2 and Lats $1 / 2$ inhibit YAP activity in the neural tube. Two possibilities may account for the lesser severity of the overproliferation phenotype as compared with that caused by YAP overexpression. First, our loss-of-function approaches might have only partially reduced the activities of endogenous kinases. Second, the amount of active YAP resulted from Mst1/2 and Lats $1 / 2$ loss of function, which relieves the inhibition on endogenous YAP, may be much less than that obtained by YAP overexpression.

Conversely, in neural tubes overexpressing Mst2, but not Mst $2^{\mathrm{KD}}$, cell death was significantly increased (Fig. $8 \mathrm{~F}, \mathrm{~J})$, whereas proliferation and differentiation appeared largely normal (data not shown). This phenotype closely resembles YAP and TEAD loss of function, in agreement with the model that Mst1/2 inhibit YAP activity through a phosphorylation-dependent event. However, one caveat is that, in addition to Lats1/2 (and hence the Hippo pathway), Mst1/2 have been shown to phosphorylate a number of substrates and lead to apoptosis (Matallanas et al. 2008). It is thus possible that the apoptosis phenotype in Mst2-transfected neural tubes was not due to YAP inhibition but rather to other Mst1/2 substrates. We therefore tested whether YAP and TEAD could rescue Mst2induced cell death. Cotransfection of TEA-VP16 and the constitutively active form of YAP $\left(\mathrm{YAP}^{\mathrm{S} 126 \mathrm{~A}}\right)$, but not wild-type YAP, with Mst2 greatly reduced the amount of cell death (Fig. 8G-J). These experiments suggest that Mst2 overexpression triggers cell death by inhibiting YAP function.

In summary, both loss-of-function and gain-of-function studies demonstrate that Mst1/2 and Lats1/2, the upstream components of the Hippo pathway, regulate neural progenitor proliferation and survival by inhibiting the activity of YAP.

\section{Discussion}

The Hippo pathway plays important roles in the control of cell proliferation and survival. Although extensively studied in fly imaginal discs, the function of this pathway during vertebrate development is largely unknown. The most critical effector of the Hippo pathway is the transcriptional coactivator Yki/YAP, which activates downstream genes via a DNA-binding transcription factor. We found that during vertebrate neural tube development, TEAD is the cognate DNA-binding factor that bridges YAP and its target genes (Fig. 8K). Furthermore, we showed that the Hippo pathway regulates the number of neural progenitor cells by affecting their proliferation, fate choice, and survival.

\section{TEAD is the DNA-binding factor of the Hippo pathway during vertebrate neural tube development}

We presented five lines of evidence demonstrating that, during neural tube development, TEAD is the DNAbinding partner of YAP. First, overexpressing YAP and a transcriptionally active form of TEAD in the neural tube both led to reduced neuronal differentiation and a marked increase in progenitor cell number. Up-regulation of cyclin D1, repression of NeuroM, accelerated pro- 

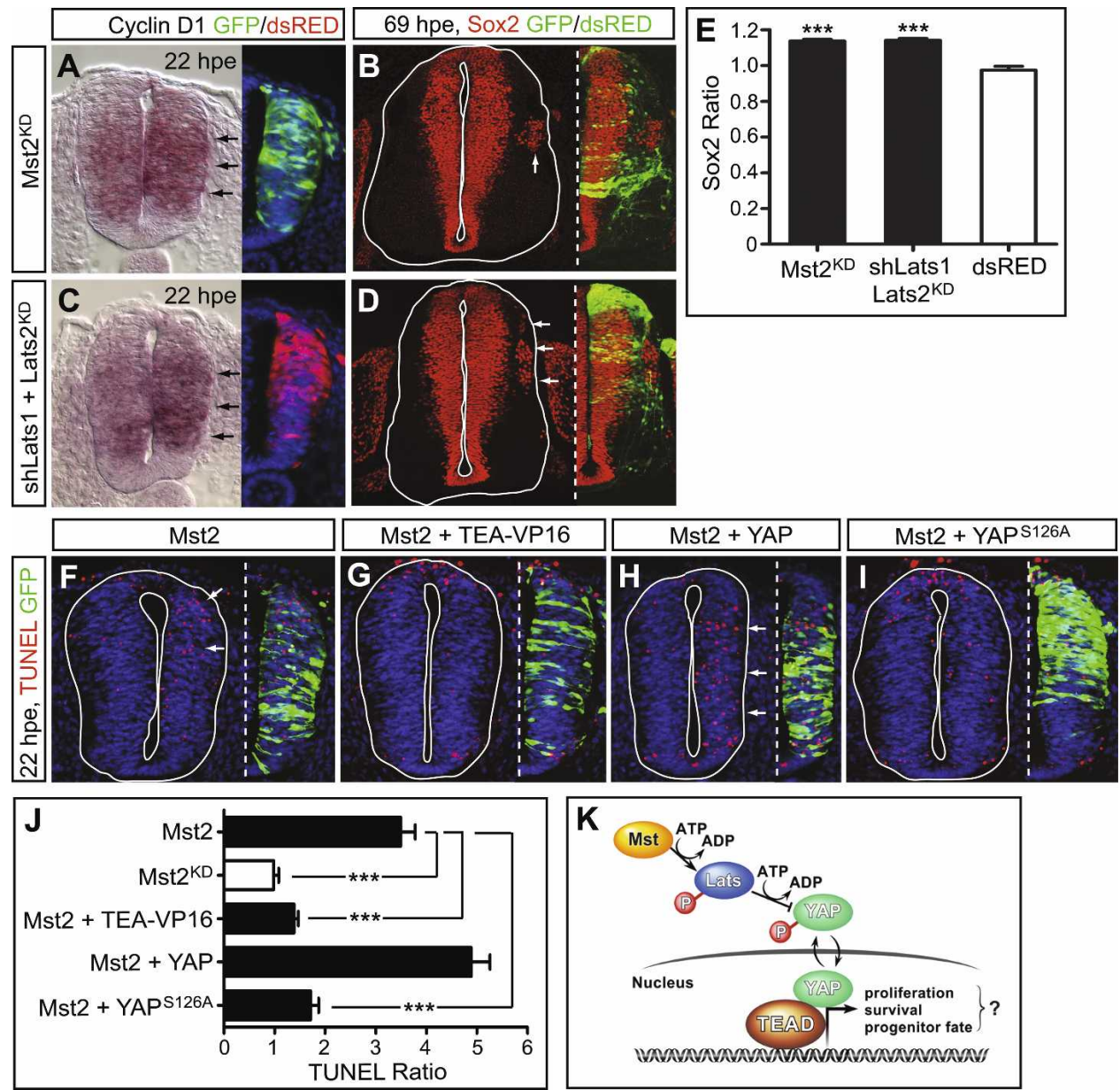

Figure 8. Mst1/2 and Lats1/2 regulate neural progenitor proliferation and survival. $(A-E)$ Inhibiting Mst $1 / 2$ with Mst $2^{\mathrm{KD}}$ and Lats $1 / 2$ with shLats1 and Lats ${ }^{\mathrm{KD}}$ caused an up-regulation of cyclin D1 expression at 22 hpe $\left(A, C\right.$, arrows) as well as a widening of the Sox $2^{+}$ progenitor zone and the appearance of ectopic Sox $2^{+}$cells in the mantle zone at 69 hpe $(B, D$, arrows). Blue signals are DAPI. (E) "Sox 2 ratio" was obtained by dividing Sox2 area in the transfected side by that in the control side. Neural tubes transfected with dsRED was used as the baseline for statistics analysis. $(F-J)$ Overexpressing Mst2 induced cell death (arrows in F). Cotransfection of TEA-VP16 and $\mathrm{YAP}^{\mathrm{S} 126 \mathrm{~A}}$, but not YAP, reduced Mst2-induced cell death. Transfected side was marked by cotransfected GFP. TUNEL ratios are Mst2: $3.5 \pm 0.3, n=5 ; \mathrm{Mst}^{\mathrm{KD}}: 0.98 \pm 0.1, n=4 ; \mathrm{Mst} 2+\mathrm{TEA}-\mathrm{VP} 16: 1.39 \pm 0.08, n=4 ; \mathrm{Mst} 2+\mathrm{YAP}: 4.9 \pm 0.4, n=5 ; \mathrm{Mst} 2+\mathrm{YAP} \mathrm{P}^{\mathrm{S} 126 \mathrm{~A}}$ : $1.7 \pm 0.2, n=5$. (K) A simplified model illustrating that the Hippo pathway regulates neural progenitor cell number through TEAD. Mst kinases phosphorylate and activate Lats kinases, which phosphorylate YAP and inhibit its nuclear translocation. Unphosphorylated YAP enters the nucleus and interacts with the DNA-binding factor TEAD. Together, they activate genes that promote proliferation, survival, and the progenitor fate.

liferation, and disruption of the neuroepithelial structure were observed in both cases. Second, YAP and TEAD loss of function also resulted in the same phenotype: increased cell death without severe defects in proliferation and differentiation. Third, repressing the downstream genes of YAP and TEAD both led to down-regulation of cyclin D1 and induction of NeuroM, resulting in cell cycle exit and neuronal differentiation. These three lines of evidence suggest that YAP and TEAD converge on the same set of target genes. The fourth line of evidence is based on a deletion within YAP that abolishes its interaction with TEAD. This mutant abrogated all of the above phenotypes, suggesting that TEAD binding is important for YAP function. Fifth, the active form of TEAD was able to rescue YAP loss of function, whereas YAP could not res- cue TEAD loss of function, indicating that TEAD is epistatic to YAP. Our finding is consistent with recent works in Drosophila and a mammalian epithelial cell line that also identified the TEAD proteins as the bridge between YAP/Yki and its target genes (Goulev et al. 2008; Wu et al. 2008; Zhang et al. 2008; Zhao et al. 2008).

\section{The vertebrate Hippo pathway regulates neural progenitor proliferation and fate choice}

We found that activating YAP and TEAD and inhibiting Mst1/2 and Lats1/2 all induced cyclin D1 expression in the neural tube, thereby promoting neural progenitor cell cycle progression. On the other hand, our data as well as others' show that constitutive expression of cy- 
clin D1 can only transiently increase neural progenitor cell number (Lobjois et al. 2008). Cells forced to express cyclin D1 undergo a few extra rounds of mitosis and, probably due to the up-regulation of cell cycle inhibitors associated with neuronal differentiation, eventually exit the cell cycle. Thus, to achieve the dramatic overproliferation phenotype, YAP and TEA-VP16 overexpression likely has induced factors that inhibit differentiation. In support of the notion that YAP and TEAD affect cell fate, we found that activating YAP/TEAD inhibits the expression of the neurogenic bHLH factor NeuroM. Conversely, repressing YAP/TEAD target genes up-regulates NeuroM and leads to premature neuronal differentiation.

Recent studies in mice also implicate the Hippo pathway in the fate choices of several progenitor cell types. Overexpressing YAP in the intestine increases the number of progenitor cells, which are maintained in an undifferentiated state until YAP expression is repressed (Camargo et al. 2007). In mice lacking WW45, the mammalian ortholog of sav, epithelial progenitors in many organs are defective in terminal differentiation (Lee et al. 2008). How the Hippo pathway governs neural progenitor fate choice remains to be determined. A number of observations suggest that the Notch and Wnt pathways may be involved. In the mouse intestine, both Notch and Wnt signalings seem to be stimulated shortly after YAP activation (Camargo et al. 2007). In Drosophila, fat and wts mutant clones as well as yki overexpression clones have increased levels of the Notch ligand Serrate in leg imaginal discs and of Wingless in wing discs (Cho et al. 2006). It is well established that the Notch pathway plays a critical role in maintaining the progenitor fate in the early neural tube. Wnt signaling has also been shown to inhibit neuronal differentiation. Excessive activation of each pathway leads to a large expansion of the neural progenitor population (Lardelli et al. 1996; Chenn and Walsh 2002; Megason and McMahon 2002), whereas blocking their signaling results in premature differentiation (Zechner et al. 2003; Hatakeyama et al. 2004).

We found that repressing YAP/TEAD target genes leads to precocious neuronal differentiation. It appears that active repression is necessary, as overexpressing the TEA domain alone-which binds to DNA but does not activate or repress transcription-was unable to do so. A similar finding was obtained with the HMG-box factors Sox1-3, which act as transcriptional activators and maintain the progenitor fate: Expression of HMG-EnR but not HMG domain alone induces differentiation (Bylund et al. 2003). Upon the initiation of neuronal differentiation, the target genes of Sox $1-3$ are repressed by Sox21, which shares the conserved HMG domains but acts as a transcriptional repressor (Sandberg et al. 2005). It is tempting to speculate that such an activator-repressor switch also operates on TEAD target genes during neurogenesis. In support of this hypothesis, we observed that some cells lateral to the Sox $2^{+}$progenitor zone appear to express TEAD1 but not YAP (Fig. 2B). These cells, judged from their location, are likely to be Neu$\mathrm{roM}^{+}$cells that have just commenced neuronal differentiation (Bylund et al. 2003). TEAD1 may interact with a transcriptional repressor in these cells to facilitate differentiation.

Neural tubes with hyperactivated YAP/TEAD manifest disorganized neuroepithelia and tumor-like rosettes, probably resulting from disruptions in adherens junctions and loss of neuroepithelial cell polarity that are observed before the onset of severe tissue dysplasia (data not shown). Similar perturbations in epithelial structure and cell polarity are found in YAP transgenic mice, WW45 mutant mice, and fly Hippo mutants (Camargo et al. 2007; Meignin et al. 2007; Polesello and Tapon 2007; Lee et al. 2008). These structural changes are reminiscent of a process known as epithelial-mesenchymal transition (EMT), in which epithelial cells lose their apical-basal polarity and adherens junctions and adopt properties that are typical of mesenchymal cells. Interestingly, overexpressing YAP in epithelial cell lines affects the expression of known EMT markers and induces EMT (Zhao et al. 2008). This activity may, at least partially, underlie the structural defects in YAP-transfected neural tubes.

\section{YAP and TEAD are required for the survival of neural} progenitor cells

Loss-of-function studies in mice have not been able to determine the roles of YAP and TEAD during neural development. YAP mutant mice are arrested around E8.5 with widespread defects (Morin-Kensicki et al. 2006), preventing an assessment of its function in neural development. TEAD proteins, with their overlapping expression in the neural tube, appear to, at least partially, compensate for each other (Chen et al. 1994; Kaneko et al. 2007; Yagi et al. 2007). We show here that inhibiting YAP and TEAD in the neural tube triggers apoptosis. Recent studies find that overexpressing YAP in the mouse liver and intestine induces several anti-apoptotic factors, including $c I A P 1$, survivin, MCL1, and Bcl- $X_{L}$ (Camargo et al. 2007; Dong et al. 2007). Moreover, diap1, the fly ortholog of $c I A P 1$, has been shown to be a direct target of Yki/SD-mediated transcription (Wu et al. 2008; Zhang et al. 2008). Thus, YAP and TEAD may directly promote neural progenitor survival. However, it is also possible that the apoptosis phenotype is secondary to other cellular defects. Identifying direct YAP/TEAD target genes in neural progenitor cells is required to address these possibilities.

\section{Mechanism of transcriptional regulation by the Hippo pathway}

We identified cyclin D1 as a potential target gene of the Hippo pathway during neural tube development. Although cyclin D1 is up-regulated by YAP/TEAD gain of function, its expression is not diminished when YAP/ TEAD function is inhibited, suggesting YAP/TEAD is not required for its basal transcription. A similar finding is made with the fly Hippo target gene expanded, whose basal expression is yki independent. However, the basal 
expression of diap1 does require yki (Wu et al. 2008; Zhang et al. 2008). Two distinct Hippo-regulated regions have been isolated from the diap1 locus. One region is responsive to yki overexpression but is largely unresponsive to the loss of yki (Wu et al. 2008), whereas the other region, although bound by Yki/SD and required for diap1 expression, does not respond to yki overexpression (Zhang et al. 2008). These observations suggest that the output of the Hippo pathway may be exquisitely sensitive to the efficacy of the signal transduction cascade: Different target genes probably respond to different thresholds of signaling activity, and, even within a target gene, different promoter elements may be tuned to respond differently.

During neural tube development, the Wnt and Shh pathways have mitogenic functions. We show here that the Hippo pathway has an opposing activity and restricts neural progenitor cells from overproliferation. It is very likely that the Hippo, Wnt, and Shh pathways interact with each other and together orchestrate the growth of the neural tube. Moreover, organ growth has to be tightly coupled to patterning. Dissecting the logic of the interplay between the growth pathways and the patterning pathways, such as Shh, BMP, retinoic acid, and Fgf pathways, will offer important insights into the mechanisms controlling nervous system development.

\section{Materials and methods}

\section{DNA constructs}

A modified pMIW vector (Muramatsu et al. 1997) was used as the backbone for all overexpression constructs. The coding sequence of every gene was PCR amplified (see Supplemental Table S1 for primers) from HH24-25 chick cDNAs. Point mutations were introduced using the QuikChange mutagenesis kit (Stratagene). shRNA constructs were generated using a chick RNAi system (Das et al. 2006). The target sequences are shYAP\#1, GCGGACTTAGCATGAGCAGTTA; shYAP\#2, CT GAGGACTATGACTACAAATA; shYAP\#1scrambled, GGAT GCAAGCGAGCCGTTTATA; shLats1, TGATCCAGTTGAT CCAGATAAA.

In ovo electroporation, immunostaining, BrdU labeling, in situ hybridization, and TUNEL assay

In ovo electroporation (see Supplemental Table S2 for plasmid concentrations) and immunostaining (see Supplemental Table S3 for antibodies) were performed as described (Megason and McMahon 2002). For BrdU labeling of HH27-28 embryos, 150 $\mu \mathrm{L}$ of $\mathrm{BrdU}(0.05 \mathrm{mg} / \mathrm{mL})$ were added at $67 \mathrm{hpe}$ onto the embryos, which were further incubated for $2 \mathrm{~h}$ at $38^{\circ} \mathrm{C}$. For cell cycle length analysis (Chenn and Walsh 2002), embryos were labeled with $50 \mu \mathrm{L}$ of $\mathrm{BrdU}$ for $30 \mathrm{~min}$ at $38^{\circ} \mathrm{C}$. Histological analyses were performed on brachial and thoracic levels of embryos harvested at 22 hpe or earlier stages and on thoracic levels of later stage embryos. For in situ hybridization, fulllength cDNAs were cloned into pBluescript SK. NeuroM probe has been described (Lee and Pfaff 2003). Probes were prepared using the Riboprobe System-T3/T7 (Promega). Hybridization was carried out according to a method described previously (Ma et al. 1996). TUNEL assay was performed with the ApopTag kit (Chemicon).
Cell culture, transfection, and coimmunoprecipitation

293T cells and chick embryonic fibroblasts (American Type Culture Collection) were cultured in DMEM medium (Invitrogen) supplemented with $10 \%$ fetal bovine serum. Cells were transfected with Lipofectamin 2000 (Invitrogen). Coimmunoprecipitation was performed as described (Cao and Sudhof 2004).

\section{Image acquisition and analysis}

Fluorescent images were acquired with a Nikon Eclipse TE300 microscope and a Bio-Rad Radiance 2100 laser scanning system. Bright field images were acquired with a Nikon Eclipse E800 microscope and a RT Slider Spot camera (Diagnostic Instruments). The ImagePro software was used for image analysis. Sections with high transfection efficiencies were used for quantifications. For Tuj1 quantification, the sum of the fluorescence intensity in the transfected region (except the lower quarter, where Tuj1 signals are very strong and tend to mask the changes in upper regions) was divided by that in the corresponding region of the control side. NF quantification was also obtained by comparing the sum of the fluorescence intensity in the transfected region with that in the corresponding untransfected region. Sox 2 and BrdU were quantified by measuring the area of immunofluorescence signals. pH3, Lim1/2, and TUNEL signals were counted manually.

\section{Acknowledgments}

We thank members of the Gage and Pfaff laboratories for advice and discussions; Chris Kintner, Ge Bai, Hoonkyo Suh, Ahmet Denli, and Stefan Aigner for critical reading of the manuscript; Jonas Muhr for Ngn2 antibody; Developmental Studies Hybridoma Bank for antibodies; Francois Guillemot and Naihe Jing for Hes1 and Hes5 in situ hybridization probes; Elizabeth Grabowski for graphics; and Mary Lynn Gage for editorial assistance. We apologize to those whose original work could not be cited due to space limitation. X.C., S.L.P., and F.H.G. are supported by NIH/NINDS.

\section{References}

Anbanandam, A., Albarado, D.C., Nguyen, C.T., Halder, G., Gao, X., and Veeraraghavan, S. 2006. Insights into transcription enhancer factor 1 (TEF-1) activity from the solution structure of the TEA domain. Proc. Natl. Acad. Sci. 103: $17225-17230$.

Azakie, A., Larkin, S.B., Farrance, I.K., Grenningloh, G., and Ordahl, C.P. 1996. DTEF-1, a novel member of the transcription enhancer factor-1 (TEF-1) multigene family. J. Biol. Chem. 271: 8260-8265.

Bylund, M., Andersson, E., Novitch, B.G., and Muhr, J. 2003. Vertebrate neurogenesis is counteracted by Sox1-3 activity. Nat. Neurosci. 6: 1162-1168.

Camargo, F.D., Gokhale, S., Johnnidis, J.B., Fu, D., Bell, G.W., Jaenisch, R., and Brummelkamp, T.R. 2007. YAP1 increases organ size and expands undifferentiated progenitor cells. Curr. Biol. 17: 1-7.

Campbell, S., Inamdar, M., Rodrigues, V., Raghavan, V., Palazzolo, M., and Chovnick, A. 1992. The scalloped gene encodes a novel, evolutionarily conserved transcription factor required for sensory organ differentiation in Drosophila. Genes \& Dev. 6: 367-379.

Cao, X. and Sudhof, T.C. 2004. Dissection of amyloid- $\beta$ precursor protein-dependent transcriptional transactivation. $J$. 
Biol. Chem. 279: 24601-24611.

Chan, E.H., Nousiainen, M., Chalamalasetty, R.B., Schafer, A., Nigg, E.A., and Sillje, H.H. 2005. The Ste20-like kinase Mst2 activates the human large tumor suppressor kinase Lats1. Oncogene 24: 2076-2086.

Chen, Z., Friedrich, G.A., and Soriano, P. 1994. Transcriptional enhancer factor 1 disruption by a retroviral gene trap leads to heart defects and embryonic lethality in mice. Genes \& Dev. 8: 2293-2301.

Chenn, A. and Walsh, C.A. 2002. Regulation of cerebral cortical size by control of cell cycle exit in neural precursors. Science 297: 365-369.

Cho, E., Feng, Y., Rauskolb, C., Maitra, S., Fehon, R., and Irvine, K.D. 2006. Delineation of a Fat tumor suppressor pathway. Nat. Genet. 38: 1142-1150.

Creasy, C.L. and Chernoff, J. 1995a. Cloning and characterization of a human protein kinase with homology to Ste20. I. Biol. Chem. 270: 21695-21700.

Creasy, C.L. and Chernoff, J. 1995b. Cloning and characterization of a member of the MST subfamily of Ste20-like kinases. Gene 167: 303-306.

Das, R.M., Van Hateren, N.J., Howell, G.R., Farrell, E.R., Bangs, F.K., Porteous, V.C., Manning, E.M., McGrew, M.J., Ohyama, K., and Sacco, M.A. 2006. A robust system for RNA interference in the chicken using a modified microRNA operon. Dev. Biol. 294: 554-563.

Dong, J., Feldmann, G., Huang, J., Wu, S., Zhang, N., Comerford, S.A., Gayyed, M.F., Anders, R.A., Maitra, A., and Pan, D. 2007. Elucidation of a universal size-control mechanism in Drosophila and mammals. Cell 130: 1120-1133.

Dyer, M.A. 2004. Mouse models of childhood cancer of the nervous system. J. Clin. Pathol. 57: 561-576.

Gotz, M. and Huttner, W.B. 2005. The cell biology of neurogenesis. Nat. Rev. Mol. Cell Biol. 6: 777-788.

Goulev, Y., Fauny, J.D., Gonzalez-Marti, B., Flagiello, D., Silber, J., and Zider, A. 2008. SCALLOPED interacts with YORKIE, the nuclear effector of the hippo tumor-suppressor pathway in Drosophila. Curr. Biol. 18: 435-441.

Hamburger, V. and Hamilton, H.L. 1992. A series of normal stages in the development of the chick embryo. 1951. Dev. Dyn. 195: 231-272.

Hatakeyama, J., Bessho, Y., Katoh, K., Ookawara, S., Fujioka, M., Guillemot, F., and Kageyama, R. 2004. Hes genes regulate size, shape and histogenesis of the nervous system by control of the timing of neural stem cell differentiation. Development 131: 5539-5550.

Howell, M., Borchers, C., and Milgram, S.L. 2004. Heterogeneous nuclear ribonuclear protein $U$ associates with YAP and regulates its co-activation of Bax transcription. J. Biol. Chem. 279: 26300-26306.

Huang, J., Wu, S., Barrera, J., Matthews, K., and Pan, D. 2005. The Hippo signaling pathway coordinately regulates cell proliferation and apoptosis by inactivating Yorkie, the Drosophila homolog of YAP. Cell 122: 421-434.

Kaneko, K.J., Kohn, M.J., Liu, C., and DePamphilis, M.L. 2007. Transcription factor TEAD2 is involved in neural tube closure. Genesis 45: 577-587.

Komuro, A., Nagai, M., Navin, N.E., and Sudol, M. 2003. WW domain-containing protein YAP associates with ErbB-4 and acts as a co-transcriptional activator for the carboxyl-terminal fragment of ErbB-4 that translocates to the nucleus. $I$. Biol. Chem. 278: 33334-33341.

Kuida, K., Haydar, T.F., Kuan, C.-Y., Gu, Y., Taya, C., Karasuyama, H., Su, M.S.S., Rakic, P., and Flavell, R.A. 1998. Reduced apoptosis and cytochrome c-mediated caspase activation in mice lacking caspase 9. Cell 94: 325-337.
Lardelli, M., Williams, R., Mitsiadis, T., and Lendahl, U. 1996. Expression of the Notch 3 intracellular domain in mouse central nervous system progenitor cells is lethal and leads to disturbed neural tube development. Mech. Dev. 59: 177-190.

Lee, S.K. and Pfaff, S.L. 2003. Synchronization of neurogenesis and motor neuron specification by direct coupling of bHLH and homeodomain transcription factors. Neuron 38: 731745.

Lee, J.H., Kim, T.S., Yang, T.H., Koo, B.K., Oh, S.P., Lee, K.P., Oh, H.J., Lee, S.H., Kong, Y.Y., Kim, J.M., et al. 2008. A crucial role of WW45 in developing epithelial tissues in the mouse. EMBO J. 27: 1231-1242.

Lobjois, V., Benazeraf, B., Bertrand, N., Medevielle, F., and Pituello, F. 2004. Specific regulation of cyclins D1 and D2 by FGF and Shh signaling coordinates cell cycle progression, patterning, and differentiation during early steps of spinal cord development. Dev. Biol. 273: 195-209.

Lobjois, V., Bel-Vialar, S., Trousse, F., and Pituello, F. 2008. Forcing neural progenitor cells to cycle is insufficient to alter cell-fate decision and timing of neuronal differentiation in the spinal cord. Neural Develop. 3: 4. doi: 10.1186/1749. 8104-3-4.

Ma, Q., Kintner, C., and Anderson, D.J. 1996. Identification of neurogenin, a vertebrate neuronal determination gene. Cell 87: 43-52.

Massague, J. 2004. G1 cell-cycle control and cancer. Nature 432: 298-306.

Matallanas, D., Romano, D., Hamilton, G., Kolch, W., and O'Neill, E. 2008. A Hippo in the ointment: MST signalling beyond the fly. Cell Cycle 7: 879-884.

McPherson, J.P., Tamblyn, L., Elia, A., Migon, E., Shehabeldin, A., Matysiak-Zablocki, E., Lemmers, B., Salmena, L., Hakem, A., Fish, J., et al. 2004. Lats2/Kpm is required for embryonic development, proliferation control and genomic integrity. EMBO J. 23: 3677-3688.

Megason, S.G. and McMahon, A.P. 2002. A mitogen gradient of dorsal midline Wnts organizes growth in the CNS. Development 129: 2087-2098.

Meignin, C., Alvarez-Garcia, I., Davis, I., and Palacios, I.M. 2007. The salvador-warts-hippo pathway is required for epithelial proliferation and axis specification in Drosophila. Curr. Biol. 17: 1871-1878.

Merkle, F.T. and Alvarez-Buylla, A. 2006. Neural stem cells in mammalian development. Curr. Opin. Cell Biol. 18: 704709.

Milewski, R.C., Chi, N.C., Li, J., Brown, C., Lu, M.M., and Epstein, J.A. 2004. Identification of minimal enhancer elements sufficient for Pax 3 expression in neural crest and implication of Tead2 as a regulator of Pax3. Development 131: 829-837.

Morin, X., Jaouen, F., and Durbec, P. 2007. Control of planar divisions by the G-protein regulator LGN maintains progenitors in the chick neuroepithelium. Nat. Neurosci. 10: 14401448.

Morin-Kensicki, E.M., Boone, B.N., Howell, M., Stonebraker, J.R., Teed, J., Alb, J.G., Magnuson, T.R., O'Neal, W., and Milgram, S.L. 2006. Defects in yolk sac vasculogenesis, chorioallantoic fusion, and embryonic axis elongation in mice with targeted disruption of Yap65. Mol. Cell. Biol. 26: 77-87.

Muramatsu, T., Mizutani, Y., Ohmori, Y., and Okumura, J.-i. 1997. Comparison of three nonviral transfection methods for foreign gene expression in early chicken embryos in ovo. Biochem. Biophys. Res. Commun. 230: 376-380.

Polesello, C. and Tapon, N. 2007. Salvador-warts-hippo signaling promotes Drosophila posterior follicle cell maturation downstream of notch. Curr. Biol. 17: 1864-1870. 
Quelle, D.E., Ashmun, R.A., Shurtleff, S.A., Kato, J.Y., Bar-Sagi, D., Roussel, M.F., and Sherr, C.J. 1993. Overexpression of mouse D-type cyclins accelerates G1 phase in rodent fibroblasts. Genes \& Dev. 7: 1559-1571.

Rakic, P. 1995. A small step for the cell, a giant leap for mankind: A hypothesis of neocortical expansion during evolution. Trends Neurosci. 18: 383-388.

Roztocil, T., Matter-Sadzinski, L., Alliod, C., Ballivet, M., and Matter, J.M. 1997. NeuroM, a neural helix-loop-helix transcription factor, defines a new transition stage in neurogenesis. Development 124: 3263-3272.

Sandberg, M., Kallstrom, M., and Muhr, J. 2005. Sox21 promotes the progression of vertebrate neurogenesis. Nat. Neurosci. 8: 995-1001.

Saucedo, L.J. and Edgar, B.A. 2007. Filling out the Hippo pathway. Nat. Rev. Mol. Cell Biol. 8: 613-621.

Srivastava, A., Simmonds, A.J., Garg, A., Fossheim, L., Campbell, S.D., and Bell, J.B. 2004. Molecular and functional analysis of scalloped recessive lethal alleles in Drosophila melanogaster. Genetics 166: 1833-1843.

St John, M.A., Tao, W., Fei, X., Fukumoto, R., Carcangiu, M.L., Brownstein, D.G., Parlow, A.F., McGrath, J., and $\mathrm{Xu}, \mathrm{T}$. 1999. Mice deficient of Lats1 develop soft-tissue sarcomas, ovarian tumours and pituitary dysfunction. Nat. Genet. 21: 182-186.

Strano, S., Munarriz, E., Rossi, M., Castagnoli, L., Shaul, Y., Sacchi, A., Oren, M., Sudol, M., Cesareni, G., and Blandino, G. 2001. Physical interaction with Yes-associated protein enhances p73 transcriptional activity. J. Biol. Chem. 276: 15164-15173.

Tao, W., Zhang, S., Turenchalk, G.S., Stewart, R.A., St John, M.A., Chen, W., and Xu, T. 1999. Human homologue of the Drosophila melanogaster lats tumour suppressor modulates CDC2 activity. Nat. Genet. 21: 177-181.

Vassilev, A., Kaneko, K.J., Shu, H., Zhao, Y., and DePamphilis, M.L. 2001. TEAD/TEF transcription factors utilize the activation domain of YAP65, a Src/Yes-associated protein localized in the cytoplasm. Genes \& Dev. 15: 1229-1241.

Walsh, C.A. 1999. Genetic malformations of the human cerebral cortex. Neuron 23: 19-29.

Wu, S., Huang, J., Dong, J., and Pan, D. 2003. hippo encodes a Ste-20 family protein kinase that restricts cell proliferation and promotes apoptosis in conjunction with salvador and warts. Cell 114: 445-456.

Wu, S., Liu, Y., Zheng, Y., Dong, J., and Pan, D. 2008. The TEAD/TEF family protein Scalloped mediates transcriptional output of the Hippo growth-regulatory pathway. Dev. Cell 14: 388-398.

Yagi, R., Chen, L.F., Shigesada, K., Murakami, Y., and Ito, Y. 1999. A WW domain-containing yes-associated protein (YAP) is a novel transcriptional co-activator. EMBO J. 18: 2551-2562.

Yagi, R., Kohn, M.J., Karavanova, I., Kaneko, K.J., Vullhorst, D., DePamphilis, M.L., and Buonanno, A. 2007. Transcription factor TEAD4 specifies the trophectoderm lineage at the beginning of mammalian development. Development 134: 3827-3836.

Zechner, D., Fujita, Y., Hulsken, J., Muller, T., Walther, I., Taketo, M.M., Crenshaw 3rd, E.B., Birchmeier, W., and Birchmeier, C. 2003. $\beta$-Catenin signals regulate cell growth and the balance between progenitor cell expansion and differentiation in the nervous system. Dev. Biol. 258: 406-418.

Zhang, L., Ren, F., Zhang, Q., Chen, Y., Wang, B., and Jiang, J. 2008. The TEAD/TEF family of transcription factor Scalloped mediates Hippo signaling in organ size control. Dev. Cell 14: 377-387.
Zhao, B., Wei, X., Li, W., Udan, R.S., Yang, Q., Kim, J., Xie, J., Ikenoue, T., Yu, J., Li, L., et al. 2007. Inactivation of YAP oncoprotein by the Hippo pathway is involved in cell contact inhibition and tissue growth control. Genes \& Dev. 21: 2747-2761.

Zhao, B., Ye, X., Yu, J., Li, L., Li, W., Li, S., Yu, J., Lin, J.D., Wang, C.Y., Chinnaiyan, A.M., et al. 2008. TEAD mediates YAP-dependent gene induction and growth control. Genes \& Dev. 22: 1962-1971. 


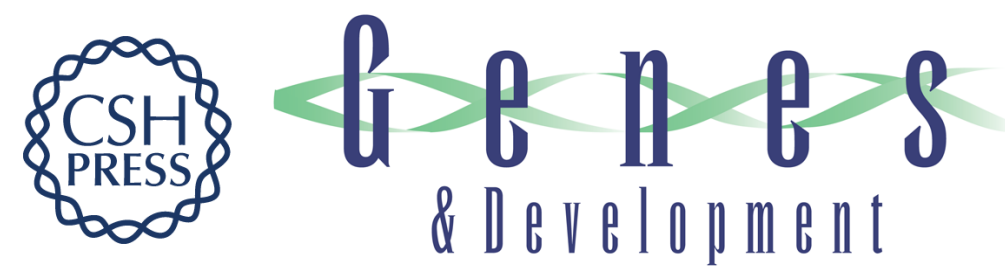

\section{YAP regulates neural progenitor cell number via the TEA domain transcription factor}

Xinwei Cao, Samuel L. Pfaff and Fred H. Gage

Genes Dev. 2008, 22: originally published online November 17, 2008

Access the most recent version at doi:10.1101/gad.1726608

\section{Supplemental http://genesdev.cshlp.org/content/suppl/2008/11/18/gad.1726608.DC1 Material}

References This article cites 60 articles, 24 of which can be accessed free at: http://genesdev.cshlp.org/content/22/23/3320.full.html\#ref-list-1

\section{License}

Email Alerting

Receive free email alerts when new articles cite this article - sign up in the box at the top Service 